\title{
Clock Genes, Metabolism, and Cardiovascular Risk
}

\author{
Roberto Tarquini, MD, ${ }^{a}$, Gianluigi Mazzoccoli, MD,*
}

\author{
KEYWORDS \\ - Clock • Gene • Circadian • Rhythm • Metabolism • Cardiovascular
}

\section{KEY POINTS}

- The biological clock rules periodic adjustments of biochemical processes controlling lipid and glucose metabolism, and the circadian timing system coordinates behavioral cycles and metabolic pathways with environmental cues.

- Metabolism, bile acid signaling, autophagy, and immunity/inflammation are driven by the clock gene machinery, which in turn is modulated by gut microbiota. Appropriate synchronization of these processes with behavioral cycles is required to thwart metabolism alteration.

- Derangements of the molecular clockwork or misalignment of the circadian timing system with respect to environmental cues causes chronodisruption and dysmetabolism, leading to cardiometabolic disease.

\section{INTRODUCTION}

The continued existence of living beings on planet Earth is taunted, especially in the wild, by environmental challenges as well as changes of ecological niches and life conditions, such as temperature swinging, food availability, and predation risk, which in turn impact processes and activities crucial for individual and species survival, such as feeding, mating, and hunting, among others. Survival advantage is warranted by proper physiologic and behavioral modifications anticipating periodic and predictable variations of the environment and cycling in a huge frequency range. ${ }^{1}$ The periodicity interval may span from the hourly variations of heart rate to the monthly and seasonal fluctuations of hormone secretion and even to the circadecennial rhythm of oscillation of umbilical cord blood parameters. ${ }^{2,3}$ The most frequent and explored biological rhythms are hallmarked by a 24-hour period of oscillation resonating with the daily transition from darkness to solar illumination dictated by Earth's rotation on its axis. This potent environmental cue is perceived by the retina via melanopsin-containing ganglion cells and transferred to the suprachiasmatic nuclei (SCN) of the hypothalamus through the glutamatergic fibers of the retino-hypothalamic tract. In mammals, 24hour rhythmicity is driven by the circadian timing system, a hierarchical multilevel organization

Conflict of Interest Statement: The authors declare that there are no conflicts of interest with respect to the authorship and/or publication of this article.

Financial Support: The study was supported by the $5 \times 1000$ voluntary contribution and by a grant (G. Mazzoccoli) through Division of Internal Medicine and Chronobiology Unit (RC1203ME46, RC1302ME31, RC1403ME50, RC1504ME53, and RC1603ME43), IRCCS Scientific Institute and Regional General Hospital "Casa Sollievo della Sofferenza", Opera di Padre Pio da Pietrelcina, San Giovanni Rotondo (F.G.), Italy.

a Department of Clinical and Experimental Medicine, School of Medicine, University of Florence, Viale Gaetano Pieraccini, 6, 50139, Florence, Italy; ${ }^{b}$ Inter-institutional Department for Continuity of Care of Empoli, School of Medicine, University of Florence, Viale Gaetano Pieraccini, 6, 50139 Florence, Italy; c Chronobiology Unit, Division of Internal Medicine, Department of Medical Sciences, IRCCS "Casa Sollievo della Sofferenza", Cappuccini Avenue, San Giovanni Rotondo, Foggia 71013, Italy

* Corresponding author.

E-mail address: g.mazzoccoli@operapadrepio.it 
composed of the SCN, composed of approximately 15,000 to 20,000 neurons in rodents ad 80,000 to 100,000 neurons in humans, working as the principal oscillator synchronizing selfsustained oscillators in the peripheral tissues by means of neural fibers of the autonomic nervous system or by humoral factors (melatonin, cortisol). ${ }^{4,5}$ Anatomic links connect the SCN to other brain regions, such as arcuate nucleus, ventromedial, dorsomedial, and lateral hypothalamic nuclei, controlling appetite, energy expenditure, and behavioral activity. 6,7 Environmental lighting is the prevailing entraining factor for SCN and sequentially for other brain areas and peripheral tissues, anyway alternative cues can overcome SCN control on peripheral clocks. In particular, feeding time is capable of disengaging peripheral oscillators and central oscillators; if experimental animals are fed only during the subjective day, when nocturnal animals usually are not active (restricted feeding), central and peripheral clocks tick in opposite phases. ${ }^{8,9}$

\section{THE MOLECULAR CLOCKWORK}

Neurons in SCN and interplaying brain areas as well as each cell in nearly all peripheral tissues harbor endowed biological clocks ticking through transcriptional-translational feedback loops (TTFLs) operated by a set of so-called clock genes and their coded proteins and revolving rhythmically with a roughly 24 -hour period. ${ }^{10-12}$ The positive limb of the TTFL in mammals, such as rodents and humans, is operated by the Period-ArntSingle-minded and basic helix-loop-helix (PASbHLH) proteins circadian locomotor output cycles kaput (CLOCK), and its paralog neuronal PAS domain protein 2 (NPAS2), and by brain and muscle aryl-hydrocarbon receptor nuclear translocatorlike/aryl-hydrocarbon receptor nuclear translocatorlike (BMAL1/ARNTL1) or its homolog BMAL2/ARNTL2. ${ }^{13}$ These transcription factors heterodimerize and bind to canonical E-box (5'CACGTG-3') cis-regulatory enhancer sequences of their target genes Period (Per1-3) and Cryptochrome (Cry 1-2). The negative limb of the TTFL is operated by PER and CRY proteins, which in turn dimerize and form a repressor complex that translocates back to the nucleus and hinders CLOCK or NPAS2/BMAL1-2 transcriptional activity. ${ }^{14,15}$ In Drosophila melanogaster as well as in other flies and insects, a cog of the molecular clockwork is represented by Timeless, which in mammals is conserved and collaborates with TIMELESS interacting protein in biological processes comprising embryonic development, cell cycle progression, DNA replication, and DNA damage response. ${ }^{16}$

\section{SIRTUINS AND THE BIOLOGICAL CLOCK}

The oscillation amplitude of numerous clock genes depends on the activity of SIRT1, a type III nicotinamide (NAM) adenine dinucleotide $\left(\mathrm{NAD}^{+}\right)$-dependent histone/protein deacetylase, which rhythmically deacetylates BMAL1, histone H3, and PER2, decreasing PER2 stability in a circadian manner. ${ }^{17,18}$ SIRT1 cofactor is de novo and cyclically synthesized from tryptophan through NAM phosphoribosyltransferase (NAMPT), the ratelimiting enzyme in the $\mathrm{NAD}^{+}$salvage pathway, whose expression is driven directly by BMAL1 with 24-hour periodicity and in the circulating form is defined as visfatin/pre-B-cell colonyenhancing factor. ${ }^{19-22}$ High NAD+ and low adenosine triphosphate levels specify low-energy status in the cell, and high adenosine monophosphate (AMP) triggers AMP-activated kinase (AMPK), which activates NAMPT and modulates the NAD+/NADH balance, ${ }^{23,24}$ working as a nutrient sensor prompted to reestablish energy balance in case of exercise, fasting, or hypoxia. ${ }^{25,26}$ SIRT1 activity is obstructed through proteinprotein interaction by deleted in breast cancer-1 (DBC1), which controls SIRT1 activity in metabolically active tissues, especially in the liver. ${ }^{27}$ DBC1 was shown in animal models fed a high-calorie diet to bind SIRT1 and impede its deacetylase activity, whereas in animals starved or fed a low-calorie diet, DBC1 remains unbound and SIRT1 activity bolsters. ${ }^{28}$ Experiments performed in vitro using cultured cells synchronized using different protocols show mitochondrial respiratory activity oscillation depends on BMAL1 levels and takes place independently from the cell type tested, the protocol of synchronization used, and the carbon source in the medium. Fluctuation in cellular $\mathrm{NAD}+$ content and clock-genes-dependent expression of NAMPT and Sirtuins 1/3 dictate the rhythmic respiratory activity and is related to the acetylation/deacetylation cycle of a single subunit of the mitochondrial respiratory chain complex I, suggesting a molecular interplay between cellular bioenergetics and the molecular clockwork operated by a dedicated interlocked transcriptionalenzymatic feedback loop. ${ }^{29,30}$

\section{POSTTRANSLATIONAL AND EPIGENETIC MODIFICATIONS}

The functioning of the molecular clockwork crucially depends on posttranslational modification of the circadian proteins, comprising phosphorylation, acetylation, sumoylation, and ubiquitination, which modulates their transcriptional activity and intracellular localization. ${ }^{31,32}$ 
Mainly, casein kinases $1-\delta$ and $1-\varepsilon$ (CK1 $\delta$ and CK1 $1 \varepsilon$ ) target Bmal1 as well as the PER and CRY proteins, tagging the latter for polyubiquitination by the E3 ubiquitin ligase complex $\beta$-transducin repeat containing protein 1 and SCF/Fbxl3 ubiquitin ligase complex (Skp1, Cullin1, F-box, and leucine-rich repeat protein 3), respectively. ${ }^{33,34}$ The AKTGSK3 $\beta$ system phosphorylates BMAL1, ${ }^{35}$ and AMPK targets the CRY proteins tagging them for degradation through the $26 \mathrm{~S}$ proteasome via the SCF/Fbxl3 ubiquitin ligase complex. ${ }^{36}$ Another layer of regulation of the biological clock depends on cyclic epigenetic modifications. CLOCK is a histone acetyltransferase (HAT) and prompts protein acetylation and chromatin remodeling holding up gene transcription. Clock joins to E-boxes in the company of cyclic adenosine monophosphate (cAMP) response element-binding protein (CBP)/ p300 and acetylates histones $\mathrm{H} 3$ and $\mathrm{H} 4$ and BMAL1, particularly the following: (1) the transcriptional coactivators and HAT p300/CBP, PCAF, and ACTR associate with CLOCK and NPAS2 to regulate trigger clock gene expression; (2) Cry2mediated hindrance of NPAS2:BMAL1 transcriptional activity is surmounted by p300 overexpression; (3) p300 shows a 24 -hour periodic association with NPAS2 in the vasculature heralding target genes expression climax; (4) a rhythm in core histone $\mathrm{H} 3$ acetylation on the mPer1 promoter in vivo correlates with mRNAs cyclical expression. ${ }^{37-39}$ Besides, 24-hour rhythms of gene transcription at the level of the whole genome are driven by cycles of histone methylation catalyzed by methyltransferase MLL3 with alternation of activating (H3K4me3) and inhibitory (H3K9me3) chromatin marks ${ }^{40}$ and cycles of histone lysine demethylation driven by the histone lysine demethylase JARID1a, which stimulates CLOCKBMAL1 heterodimer transcriptional activity. ${ }^{41}$

\section{AN AUXILIARY LOOP IN THE MOLECULAR CLOCKWORK}

The oscillation of the starting cog of the TTFLpositive limb depends on the cycling of the reverse transcript of the erythroblastosis gene (REV-ERB) $\alpha / \beta$ and the retinoic acid-related (RAR) orphan receptor (ROR) $\alpha, \beta / \delta, \gamma$, whose expression is driven by the molecular clockwork and hard-wires an additional regulatory loop controlling BMAL1 expression. REV-ERB $\alpha / \beta$, not capable of engaging coactivators and trigger target gene transcription, binds ROR-specific response elements (RORE) in Bmal1, Clock, and Cry1 promoters, impeding binding and activation of transcription by ROR $\alpha .{ }^{42-44} \mathrm{ROR} \alpha$ cooperates with the transcriptional coactivator peroxisome proliferator-activated receptor (PPAR) $\gamma$ coactivator-1 $\alpha$ (PGC-1 $\alpha$ ), which engages chromatinremodeling complexes to the proximal Bmal1 promoter and prompts Bmal1 transcription. Conversely, REV-ERB $\alpha$ cooperates with histone deacetylase 3 (HDAC3) and nuclear receptor corepressor 1 (NCOR1), operates as an HDAC3 activating subunit and induces repression of transcription. ${ }^{45}$ As a result, the 24-hour rhythm of the ROR $\alpha / P G C-1 \alpha$ activator complex and REV$\mathrm{ERB} \alpha / \mathrm{NCOR} 1-\mathrm{HDAC} 3$ repressor complex recruitment manages the 24-hour rhythmicity of Bmal1 expression. Experiments performed in mouse models showed that HDAC3 binds to the liver genome with circadian periodicity and drives the expression of gene-enriching pathways involved in lipid metabolism, whose alteration induces in vivo hepatic steatosis. ${ }^{46}$ Heme is a physiologic ligand of REV-ERB $\alpha / \beta$, binds to the ligandbinding domain with a 1:1 stoichiometry, and enhances thermal stability of these nuclear receptors. Heme synthesis is catalyzed by the ratelimiting enzyme delta-aminolevulinate synthase 1 , whose expression is driven by the molecular clockwork; its binding to REV-ERBs induces corepressor NCOR1 recruitment, with repression of target genes (Bmal1 included), whereas heme dissociation prompts the expression of target genes according to modifications in intracellular redox balance. ${ }^{47,48}$ REV-ERBs are also highly responsive to the redox state and gases. The addition of nitric oxide pulls out transcription repression induced by heme-bound REVERBs. ${ }^{49}$ In addition, a thiol-disulfide redox switch modulates heme and REV-ERB $\beta$ interaction; the reduced dithiol state of REV-ERB $\beta$ binds heme 5-fold more tightly than the oxidized disulfide state. However, changes in the iron redox state do not impact heme binding to the ligand binding domain. ${ }^{50}$ Moreover, heme influences BMAL1NPAS2 transcription activity in vitro through a NPAS2 heme-binding motif via inhibition of DNA binding in response to carbon monoxide. ${ }^{51}$

\section{THE CLOCK-CONTROLLED GENES}

The molecular clockwork drives the expression of so-called clock-controlled genes, some in common among the different peripheral tissues and others specific to particular tissues and defined output genes, which manage biological processes at the cell level and physiologic functions at the tissue and organ level. ${ }^{52}$ Principally, CLOCK:BMAL1 heterodimer drives the expression of the prolineand acidic amino acid-rich domain basic leucine zipper transcription factors albumin gene D-site binding protein (DBP), thyrotroph embryonic 
factor, and hepatic leukemia factor (HLF); these sequentially drive the transcription of thousands of genes. ${ }^{53}$ In turn, DBP triggers Per1 transcription feeding back on the molecular clockwork, ${ }^{54}$ whereas REV-ERBs bind to RORE and trigger the expression of the nuclear factor interleukin 3 regulated protein (defined adenoviral E4 proteinbinding protein, [E4BP4] as well), which fluctuates in antiphase regarding DBP, in order that these transcription factors manage the expression of downstream genes peaking in an opposite phase. ${ }^{55}$ Another cog of the machinery is represented by the bHLH transcription factors differentially expressed in chondrocytes protein 1 (DEC1) and 2 (DEC2), which manage transcriptional repression/regulation of multiple circadian genes and feed back to the molecular clockwork. ${ }^{56,57}$

\section{THE BIOLOGICAL CLOCK AND THE NUCLEAR RECEPTORS}

The clock gene machinery drives rhythmic oscillations of several biological processes whose output feeds back in the biological clock (Fig. 1). A crucial role in this interplay is played by the nuclear receptors, ligand-dependent transcription factors capable of binding lipophilic ligands and interact directly with promoters of specific DNA sequences to modulate target gene expression. The intracellular level of several nuclear receptors oscillate with circadian rhythmicity in several metabolically active tissues, such as liver, muscle, and adipose tissue and in particular include constitutive androstane receptor (CAR); estrogen-related receptor (ERR) $\alpha, \beta$, and $\gamma$; farnesoid receptor (FXR) $\alpha$ and $\beta$; glucocorticoid receptor (GR); Nur-related protein 1; PPAR $\alpha, \delta / \beta$, and $\gamma ; \operatorname{RAR} \alpha, \beta$, and $\gamma$; retinoid $X$ receptors (RXR) $\alpha, \beta$, and $\gamma$; small heterodimeric partner (SHP); and thyroid hormone receptor $\alpha^{58,59}$ The rhythmic assembly of metabolites capable of binding nuclear receptors and the interplay among molecular clockworks and signaling pathways activated on ligand binding to nuclear receptors sustain the circadian regulation of metabolism. The biological clock drives the oscillation of nuclear receptors as well as their ligands; sequentially, the nuclear receptors gauge the metabolic status and feed back in the clock gene machinery binding to response elements on definite clock genes, specifying transcriptional networks that convey time-related and feeding-related cues to the metabolic pathways. ${ }^{60}$ For instance, oxysterols and bile acids are produced with 24-hour periodicity, bind liver $X$ receptor (LXR) and FXR, respectively, and in turn oxysterols binding LXRs trigger, whereas bile acids binding FXRs hinder the expression of Cyp7a1, which encodes CYP7A1, the ratelimiting enzyme in bile acid synthesis whose expression oscillates with circadian rhythmicity driven by the alternate binding of LXR and FXR on response elements at the gene promoter level. ${ }^{61,62}$

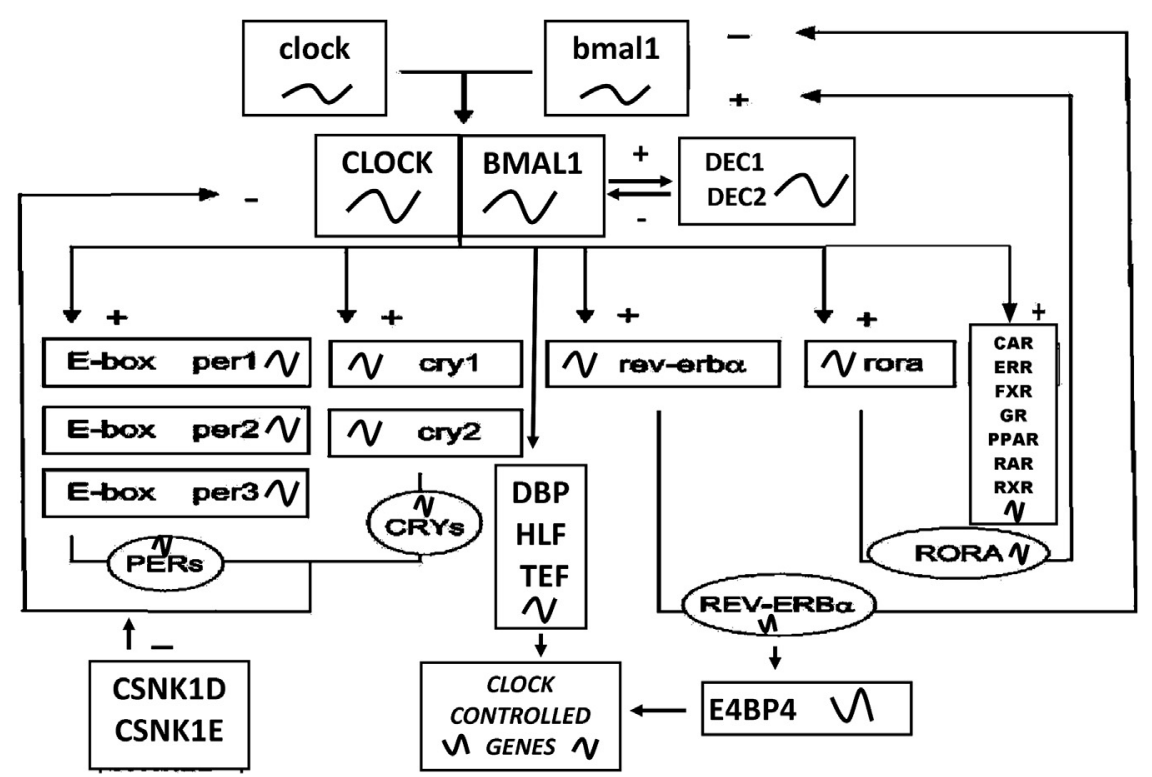

Fig. 1. The functioning of the clock gene machinery. Plus signs indicate activation; minus signs indicate inhibition; arrow-ended continuous lines indicate molecular interaction. CAR, constitutive androstane receptor; ERR, estrogen-related receptor; FXR, farnesoid receptor; GR, glucocorticoid receptor; RXR, retinoid X receptors. 


\section{THE MOLECULAR CLOCKWORK AND INTERMEDIATE METABOLISM}

The biological clock drives the expression of many genes enriching the metabolic pathways that manage intermediate metabolism and coordinates the enzymatic cascades implicated in lipid and glucose metabolism. ${ }^{63}$

\section{Lipid Metabolism}

The circulating levels of lipids and the activity of enzymes catalyzing their synthesis and lysis, such as 3-hydroxy-3-methylglutaryl coenzyme A (HMG-CoA) reductase, fatty acid synthase, fatty acyl-CoA synthetase 1 , as well as the carriers involved in their transport, such as apolipoprotein A-IV and C-III, fatty acid transport protein 1, or the receptors mediating their turnover, such as low-density lipoprotein receptor, oscillate with circadian rhythmicity in mammals. ${ }^{63}$ The 24-hour cycle of Cyp7a1 expression is additionally controlled by REV-ERB $\alpha$, DBP/E4BP4, and DEC2, which manage correct ruling of the circadian pattern, working through Rev-ROR response elements, DBP/E4BP4-binding elements, and Eboxes, respectively. ${ }^{64} \mathrm{ROR} \alpha$ is capable of binding cholesterol and its metabolites, for example, 7oxygenated sterol, with transcriptional activity modulation, ${ }^{65}$ and triggers the expression of apoC-III, a very low-density lipoprotein component, ${ }^{66}$ whose transcription is thwarted by REVERB $\alpha .{ }^{67}$ On its side, REV-ERB $\alpha$ drives sterol regulatory element binding protein (SREBP) activity controlling the 24-hour rhythm of oscillation of the insulin-induced gene (INSIG) 2, which encodes an enzyme that seizes at the level of the endoplasmic reticulum membranes, the SREBcleavage activating protein-INSIG-SREBP complex, which gauges cholesterol accessibility; in addition, driving the rhythmic nuclear accrual of SREBP, REV-ERB $\alpha$ also drives the time-related expression of $\mathrm{Hmgcr}$, encoding HMG-CoA reductase, the rate-limiting enzyme of the mevalonate pathway, involved in the biosynthesis of cholesterol and other isoprenoids. ${ }^{68}$ Besides, REV$\mathrm{ERB} \alpha$ manages bile acid metabolism via fluctuations of oxysterol synthesis and LXR activity and interplays with FXR to regulate SHP, hinders the expression of SHP and E4BP4 expression and triggers the expression of Cyp7a1 in the liver. ${ }^{69}$ REV-ERB $\alpha$ manages lipid metabolism also through epigenetic changes brought on via HDAC3-NCOR1 complex recruitment at the level of genes involved in lipid metabolism, causing chromatin remodeling and histone modification: during the activity/feeding time, small REV-ERB $\alpha$ levels decrease HDAC3 binding to the liver genome and allow lipid buildup; however, during the resting/fasting time, high REV-ERB $\alpha$ levels augment HDAC3 recruitment to liver metabolic genes, hampering lipid biosynthesis. ${ }^{46}$

\section{Glucose Metabolism}

Glucose levels must be accurately gauged to provide a vital energy supply for cells in the different tissues of the organism and the equilibrium between glycogen anabolism and catabolism in metabolically active tissues helps to maintain roughly stable concentrations in the peripheral blood during the 24-hour day. On food intake, increasing plasma glucose levels prompts insulin secretion by Langerhans islets $\beta$ cells in the pancreas, insulin signaling pathway triggering, glucose uptake, and polymerization into glycogen stores. However, glycogenolysis and/or gluconeogenesis in the liver during fasting generates glucose and the increase of GLUT2 expression induces GLUT2-mediated transport of glucose in the peripheral blood. Circadian changes ruled by central and peripheral biological oscillators drive the transcription of genes encoding enzymes and carriers implicated in glucose metabolism, comprising glycogen synthase 2 (GYS2), glycogen phosphorylase, phosphoenolpyruvate carboxykinase (PEPCK), glucokinase, glucose-6phosphatase (GLC-6-Pase), and the glucose transporter GLUT2, among the others. ${ }^{70,71}$ The biological clock drives glucose metabolism and in particular CRY1 and CRY2 control gluconeogenesis in the liver decreasing CAMP signaling in response to $G$ protein-coupled receptor activation, ${ }^{72}$ CLOCK drives glycogen synthesis in the liver triggering Gys2 transcription, ${ }^{73}$ KLF10, a transcription factor encoded by a clock-controlled gene, hinders hepatic glucose production decreasing Pepck expression. ${ }^{74}$ Glucocorticoids control glucose homeostasis and circadian rhythmicity modulating the expression of core clock genes, specifically Per1 and Per2, through binding via GRs to glucocorticoid response elements in their promoters. ${ }^{75,76} \mathrm{GR}$ levels oscillate with 24hour periodicity in metabolically active tissues, in particular in white and brown adipose tissue, linking time-related and feeding-related cues to manage synchronicity between metabolic adjustments and clock gene machinery as well as activity and feeding. ${ }^{76}$ Furthermore, Rev-erb $\alpha$ inhibits gluconeogenic gene expression in the liver and modulates hepatic glucose production in response to heme, ${ }^{77}$ whereas $\mathrm{ROR} \alpha$ induces the expression of GLC-6-Pase and adjusts glycogen metabolism in the liver. ${ }^{78}$ Specifically, the SWI/ SNF chromatin-remodeling complex subunit 
BAF60a, expressed in mouse liver with circadian rhythmicity, is bound at ROR response elements on the proximal Bmal1 and G6Pc promoters, prompts their transcription through coactivation of $R O R \alpha$, and impacts the harmonized regulation of circadian clock, glucose metabolism, and energy homeostasis in the liver. ${ }^{79}$ Moreover, experiments performed in primary hepatocytes showed that PGC- $1 \alpha$ physically interacts with $\mathrm{CK} 1 \delta$ and is phosphorylated at multiple sites within its arginine/serine-rich domain and sequentially degraded through the proteasome system, with the decrease of transcription of genes enriching pathways involved in hepatic gluconeogenesis and glucose secretion. ${ }^{80}$

\section{THE BIOLOGICAL CLOCK AND DERANGED METABOLISM}

The several cogs of the biological clock manage signaling pathways and biochemical reactions crucially involved in metabolic regulation and alteration of the molecular clockwork severely impacts lipid and glucose homeostasis. A comprehensive revision of the scientific literature regarding the role played by the altered functioning of the molecular clockwork in the derangement of lipid metabolism and in particular in liver steatosis, the most important anatomopathological manifestation of metabolic syndrome, is provided by a previous review article. ${ }^{81}$ Regarding the role played by the molecular clockwork in glucose metabolism, its crucial involvement is corroborated by the reduced rhythmicity of clock genes expression found in peripheral leukocytes of patients affected by type 2 diabetes $^{82}$ and by the increased risk of impaired fasting glucose and type 2 diabetes highlighted by genome-wide association studies in subjects carrying a Cry2 variant allele. ${ }^{83,84}$ Accordingly, experiments performed in mouse models showed that Clock and Bmal1 mutation induces disrupted glucose homeostasis. ${ }^{85}$ Clock $^{\Delta 19 / 419}$ and pancreas-specific Bmal1 ${ }^{-1-}$ mutant mice are hallmarked by altered glucose tolerance, decreased insulin secretion, and reduced size and proliferation of pancreatic islets deteriorating in the course of time..$^{86,87}$ Furthermore, Langerhans islets $\beta$ cells harbor a self-sustained and autonomous molecular clockwork, ${ }^{88}$ and disruption of the biological clock induced altered transcription of genes enriching pathways involved in insulin secretion (GNAQ, ATP1A1, ATP5G2, KCNJ11) as well as granule maturation and release (VAMP3, STX6, SLC30A8) and caused altered circadian pattern of basal insulin secretion by human islet cells synchronized in vitro. ${ }^{89}$ Besides, mice with specific Bmal1 disruption in the liver are hallmarked by deranged hepatocyte molecular clockwork; GLUT2 expression is stably low; these animal models show undue glucose clearance, altered rhythmic patterns of hepatic glucose regulatory genes expression, and hypoglycemia expression during the fasting/resting phase of the nychthemeral period. ${ }^{90}$

\section{THE CLOCK GENE MACHINERY, LIVER STEATOSIS, AND CARDIOVASCULAR DISEASE}

The altered functioning of the biological clock is critically involved in the pathogenesis of nonalcoholic fatty liver disease (NAFLD), which is associated with increased risk of cardiovascular disease. ${ }^{91}$ NAFLD is the most frequent hepatic pathology in the Western world ${ }^{92}$ and in one-fifth of all cases may progress to chronic hepatic inflammation (nonalcoholic steatohepatitis [NASH]) associated with cirrhosis, portal hypertension, and hepatocellular carcinoma. ${ }^{93}$ Diseases related to dysmetabolism represent public health problems and pose a huge economic and social burden on national health systems worldwide. Obesity, a distinctive metabolic syndrome trait, augments the risk for diabetes and cardiovascular diseases; NAFLD is considered the hepatic manifestation of metabolic syndrome. ${ }^{94}$ Impressive modifications occurred throughout the previous decades in dietary macronutrient intake, such as overconsumption of energy-dense foods, particularly high-fat and high-sugar diets, which, in addition to reduced physical activity, determine energy imbalance leading to obesity and impacted metabolic diseases prevalence. ${ }^{95}$ Nutrient-sensing information is exchanged among organs to preserve systemic energy homeostasis, and the liver plays a key role in the integration and processing of signals derived from other tissues, such as intestine, pancreas, and adipose tissue. Interorgan communication is conveyed by humoral factors, such as insulin, adipocytokines, and glucocorticoids; by the autonomic nervous system ${ }^{96}$; and by dietary signals, such as fatty acids, glucose, and other metabolites: these factors are sensed by nuclear receptors that consecutively control nutrient signaling pathways. Bile acids, chiefly identified as important detergents necessary for lipid absorption in the intestine, are able to turn on nuclear receptor signaling pathways and come out as crucial metabolism regulators. ${ }^{60}$ In the past decade, an everincreasing bulk of evidence has highlighted a critical role played by the intestine in molecular regulation of diet-related diseases that exceeds its function in nutrient digestion and extraction to maintain body metabolic homeostasis. The intestine secretes enteroendocrine hormones as well, 
and the harbored gut microbial flora is ever more regarded as an essential player in the modulation of metabolic processes. Consequently, innovative preventive and therapeutic approaches, for instance, drugs targeting nuclear receptors, bile acid signaling, or gut microbiota modulation, are investigated in addition to conventional strategies, including diet and physical activity, which have been ineffective in diminishing metabolic disease prevalence. $^{97}$

\section{THE BIOLOGICAL CLOCK AT THE CROSSROAD OF AUTOPHAGY, GUT MICROBIOTA, INFLAMMATION, BILE ACID SIGNALING, AND INTERMEDIATE METABOLISM}

Multifaceted interactions occur among metabolic pathways of lipids, glucose, bile acids and autophagy, inflammation, and their regulation by the biological clock in response to nutrients, bile acids, hormones, nuclear receptors, or gut microbiota. ${ }^{98-103}$ This interplay is supported by strong evidence: (1) the metabolic derangements underlying NAFLD and the progression from NAFLD to $\mathrm{NASH}$ hint of a key role played by nutrients and bile acids acting as ligands of nuclear receptors, which manage the metabolic pathways, and as signaling molecules in metabolism and inflammation. Bile acids influence macrophage function, energy homeostasis, and gastrointestinal insulinotropic hormones secretion and are metabolized by the gut microbiota, which may change the bile acids binding capacity of their receptors and influence the intestinal immune system and the metabolic processes ${ }^{104-109}$; (2) NLRP6 and NLRP3 inflammasomes negatively regulate NAFLD to $\mathrm{NASH}$ progression through changes of the gut microbiota configuration and entry of Toll-like receptor (TLR) 4 and TLR9 agonists into the portal circulation, with modulation of hepatic tumornecrosis factor (TNF)- $\alpha$ expression driving NASH progression, and TLR7 impacts NAFLD pathogenesis as well ${ }^{110,111}$; (3) recent studies have pinpointed the role played by autophagy in NAFLD pathogenesis, suggesting the therapeutic potential of its regulation. ${ }^{112}$ Remarkably, hepatic metabolic pathways and bile acid synthesis as well as autophagic and immune/inflammatory processes are driven by the biological clock. Besides, gut microbiota impact the biological clock $^{113}$; appropriate timing of circadian patterns of hormone secretion, metabolism, bile acid turnover, autophagy, and inflammation with behavioral cycles is necessary to avoid hepatic dysfunction and metabolic disorders. ${ }^{114-123}$ Furthermore, experiments performed in aryl hydrocarbon receptor (AHR, a xenobiotic receptor for exogenous toxicants) liver-specific and inducible transgenic mice fed an obesogenic diet showed that AHR signaling pathway activation worsens liver steatosis and in contradiction avoids obesity and systemic insulin resistance interplaying with the biological clock. ${ }^{124,125}$ Interestingly, fibroblast growth factor 21, which interacting with $\beta$-Klotho coordinates a change to oxidative metabolism during fasting and starvation and has been involved as a mediator joining nutrition, growth, reproduction, and longevity, ${ }^{126}$ was recognized as a direct AHR target in the liver and as the inducer of the systemic metabolic benefits in addition to liver steatosis observed in AHR transgenic mice. ${ }^{124,125}$

\section{SUMMARY}

The biological clock controls the molecular signaling pathways involved in metabolism regulation; the circadian timing system drives sleep/ wake, rest/activity, and fasting/feeding rhythmicity, harmonizing behavioral cycles with energy flux and expenditure and synchronizing the timing of anabolic/catabolic processes with environmental cues, predominantly light/dark alternation and temperature fluctuations. The cogs of the molecular clockwork drive the periodic oscillation of biochemical processes; the nuclear receptors sense nutrient levels and the cellular redox state, guiding the recruitment of coactivators, corepressors, HATs, and HDACs to DNA sequences. These molecular events prompt chromatin remodeling and histone modifications and trigger rhythms of epigenetic modification, transcriptional activity, and gene expression, coordinating metabolic pathways with nychthemeral rhythmicity of behavior. Alteration of the biological clock as well as misalignment of body 24-hour rhythmicity with respect to environmental cues lead to chronodisruption with internal synchronization failure and metabolic derangements, ultimately causing liver steatosis, obesity, metabolic syndrome, and diabetes mellitus, with increased risk of cardiovascular diseases.

\section{REFERENCES}

1. Dunlap JC. Molecular bases for circadian clocks. Cell 1999;96(2):271-90.

2. Mazzoccoli G, Miscio G, Fontana A, et al. Time related variations in stem cell harvesting of umbilical cord blood. Sci Rep 2016;6:21404.

3. Scholkmann F, Miscio G, Tarquini R, et al. The circadecadal rhythm of oscillation of umbilical cord blood parameters correlates with geomagnetic activity - an analysis of long-term measurements (1999-2011). Chronobiol Int 2016;33(9):1136-47. 
4. Albrecht U. Timing to perfection: the biology of central and peripheral circadian clocks. Neuron 2012;74(2):246-60.

5. Nagoshi E, Saini C, Bauer C, et al. Circadian gene expression in individual fibroblasts: cellautonomous and self-sustained oscillators pass time to daughter cells. Cell 2004;119:693-705.

6. Kalsbeek A, Palm IF, La Fleur SE, et al. SCN outputs and the hypothalamic balance of life. J Biol Rhythms 2006;21(6):458-69.

7. Luo AH, Aston-Jones G. Circuit projection from suprachiasmatic nucleus to ventral tegmental area: a novel circadian output pathway. Eur J Neurosci 2009;29(4):748-60.

8. Damiola F, Le Minh N, Preitner N, et al. Restricted feeding uncouples circadian oscillators in peripheral tissues from the central pacemaker in the suprachiasmatic nucleus. Genes Dev 2000;14: 2950-61.

9. Stokkan KA, Yamazaki S, Tei H, et al. Entrainment of the circadian clock in the liver by feeding. Science 2001;291:490-3.

10. Mazzoccoli G, Francavilla M, Pazienza V, et al. Differential patterns in the periodicity and dynamics of clock gene expression in mouse liver and stomach. Chronobiol Int 2012;29(10):1300-11.

11. Mazzoccoli G, Francavilla M, Giuliani F, et al. Clock gene expression in mouse kidney and testis: analysis of periodical and dynamical patterns. J Biol Regul Homeost Agents 2012;26(2):303-11.

12. Bonny $\mathrm{O}$, Vinciguerra M, Gumtz ML, et al. Molecular bases of circadian rhythmicity in renal physiology and pathology. Nephrol Dial Transplant 2013;28(10):2421-31.

13. Mazzoccoli G, Rubino R, Tiberio C, et al. Clock gene expression in human and mouse hepatic models shows similar periodicity but different dynamics of variation. Chronobiol Int 2016;33(2):181-90.

14. $\mathrm{Ko} \mathrm{CH}$, Takahashi JS. Molecular components of the mammalian circadian clock. Hum Mol Genet 2006; 15(Spec No. 2):R271-7.

15. Lowrey PL, Takahashi JS. Genetics of circadian rhythms in mammalian model organisms. Adv Genet 2011;74:175-230.

16. Mazzoccoli G, Laukkanen $\mathrm{MO}$, Vinciguerra M, et al. A timeless link between circadian patterns and disease. Trends Mol Med 2016;22(1):68-81.

17. Nakahata Y, Kaluzova M, Grimaldi B, et al. The NAD+-dependent deacetylase SIRT1 modulates CLOCK-mediated chromatin remodeling and circadian control. Cell 2008;134(2):329-40.

18. Asher G, Gatfield D, Stratmann M, et al. SIRT1 regulates circadian clock gene expression through PER2 deacetylation. Cell 2008;134(2):317-28.

19. Nakahata Y, Sahar S, Astarita G, et al. Circadian control of the NAD+ salvage pathway by CLOCK-SIRT1. Science 2009;324:654-7.
20. Ramsey K, Yoshino J, Brace CS, et al. Circadian clock feedback cycle through NAMPT-mediated NAD+ biosynthesis. Science 2009;324:651-4.

21. Sahar S, Nin V, Barbosa MT, et al. Altered behavioral and metabolic circadian rhythms in mice with disrupted NAD+ oscillation. Aging (Albany NY) 2011;3(8):794-802.

22. Benedict C, Shostak A, Lange T, et al. Diurnal rhythm of circulating nicotinamide phosphoribosyltransferase (Nampt/visfatin/PBEF): impact of sleep loss and relation to glucose metabolism. J Clin Endocrinol Metab 2012;97(2):E218-22.

23. Fulco M, Sartorelli V. Comparing and contrasting the roles of AMPK and SIRT1 in metabolic tissues. Cell Cycle 2008;7:3669-79.

24. Cantó C, Auwerx J. PGC-1alpha, SIRT1 and AMPK, an energy sensing network that controls energy expenditure. Curr Opin Lipidol 2009;20:98-105.

25. Kahn BB, Alquier T, Carling D, et al. AMP-activated protein kinase: ancient energy gauge provides clues to modern understanding of metabolism. Cell Metab 2005;1(1):15-25.

26. Long YC, Zierath JR. AMP-activated protein kinase signaling in metabolic regulation. J Clin Invest 2006;116(7):1776-83.

27. Kim JE, Chen J, Lou Z. DBC1 is a negative regulator of SIRT1. Nature 2008;451(7178):583-6.

28. Escande C, Chini CC, Nin V, et al. Deleted in breast cancer-1 regulates SIRT1 activity and contributes to high-fat diet-induced liver steatosis in mice. J Clin Invest 2010;120(2):545-58.

29. Cela O, Scrima R, Pazienza V, et al. Clock genesdependent acetylation of complex I sets rhythmic activity of mitochondrial OxPhos. Biochim Biophys Acta 2016;1863(4):596-606.

30. Scrima R, Cela O, Merla G, et al. Clock-genes and mitochondrial respiratory activity: evidence of a reciprocal interplay. Biochim Biophys Acta 2016; 1857(8):1344-51.

31. Lee J, Lee $Y$, Lee MJ, et al. Dual modification of BMAL1 by SUMO2/3 and ubiquitin promotes circadian activation of the CLOCK/BMAL1 complex. Mol Cell Biol 2008;28(19):6056-65.

32. Eide EJ, Vielhaber EL, Hinz WA, et al. The circadian regulatory proteins BMAL1 and cryptochromes are substrates of casein kinase $\mid \varepsilon$. J Biol Chem 2002; 277:17248-54.

33. Agostino PV, Harrington ME, Ralph MR, et al. Casein kinase-1-epsilon (CK1epsilon) and circadian photic responses in hamsters. Chronobiol Int 2009;26:126-33.

34. Sahar S, Zocchi L, Kinoshita C, et al. Regulation of BMAL1 protein stability and circadian function by GSK3beta-mediated phosphorylation. PLoS One 2010;5(1):e8561.

35. Lamia KA, Sachdeva UM, Ditacchio L, et al. AMPK regulates the circadian clock by cryptochrome 
phosphorylation and degradation. Science 2009; 326:437-40.

36. Cardone L, Hirayama J, Giordano F, et al. Circadian clock control by SUMOylation of BMAL1. Science 2005;309(5739):1390-4.

37. Curtis AM, Seo SB, Westgate EJ, et al. Histone acetyltransferase-dependent chromatin remodeling and the vascular clock. J Biol Chem 2004; 279(8):7091-7.

38. Doi M, Hirayama J, Sassone-Corsi P. Circadian regulator CLOCK is a histone acetyltransferase. Cell 2006;125:497-508.

39. Hirayama J, Sahar S, Grimaldi B, et al. CLOCKmediated acetylation of BMAL1 controls circadian function. Nature 2007;450:1086-90.

40. Valekunja UK, Edgar RS, Oklejewicz M, et al. Histone methyltransferase MLL3 contributes to genome-scale circadian transcription. Proc Natl Acad Sci U S A 2013;110(4):1554-9.

41. Di Tacchio L, Le HD, Vollmers C, et al. Histone lysine demethylase JARID1a activates CLOCKBMAL1 and influences the circadian clock. Science 2011;333(6051):1881-5.

42. Preitner N, Damiola F, Lopez-Molina L, et al. The orphan nuclear receptor REV-ERBalpha controls circadian transcription within the positive limb of the mammalian circadian oscillator. Cell 2002; 110:251-60.

43. Burris TP. Nuclear hormone receptors for heme: REV-ERBalpha and REV-ERBbeta are ligandregulated components of the mammalian clock. Mol Endocrinol 2008;22(7):1509-20.

44. Mazzoccoli G, Cai Y, Liu S, et al. REV-ERBalpha and the clock gene machinery in mouse peripheral tissues: a possible role as a synchronizing hinge. J Biol Regul Homeost Agents 2012;26(2):265-76.

45. Alenghat T, Meyers K, Mullican SE, et al. Nuclear receptor corepressor and histone deacetylase 3 govern circadian metabolic physiology. Nature 2008;456:997-1000.

46. Feng D, Liu T, Sun Z, et al. A circadian rhythm orchestrated by histone deacetylase 3 controls hepatic lipid metabolism. Science 2011;331(6022): 1315-9.

47. Kaasik K, Lee CC. Reciprocal regulation of haem biosynthesis and the circadian clock in mammals. Nature 2004;430(6998):467-71.

48. Raghuram S, Stayrook KR, Huang P, et al. Identification of heme as the ligand for the orphan nuclear receptors REV-ERBalpha and REV-ERBbeta. Nat Struct Mol Biol 2007;14(12):1207-13.

49. Pardee KI, Xu X, Reinking J, et al. The structural basis of gas-responsive transcription by the human nuclear hormone receptor REV-ERBbeta. PLoS Biol 2009; $7(2): e 43$.

50. Gupta N, Ragsdale SW. Thiol-disulfide redox dependence of heme binding and heme ligand switching in nuclear hormone receptor rev-erb \{beta\}. J Biol Chem 2011;286(6):4392-403.

51. Gilles-Gonzalez MA, Gonzalez G. Signal transduction by heme-containing PAS-domain proteins. J Appl Physiol (1985) 2004;96(2):774-83.

52. Bozek K, Relógio A, Kielbasa SM, et al. Regulation of clock-controlled genes in mammals. PLoS One 2009; 4(3):e4882.

53. Gachon F, Olela FF, Schaad O, et al. The circadian PAR-domain basic leucine zipper transcription factors DBP, TEF, and HLF modulate basal and inducible xenobiotic detoxification. Cell Metabol 2006;4:25-36.

54. Yamaguchi S, Mitsui S, Yan L, et al. Role of DBP in the circadian oscillatory mechanism. Mol Cell Biol 2000;20(13):4773-81.

55. Mitsui S, Yamaguchi S, Matsuo T, et al. Antagonistic role of E4BP4 and PAR proteins in the circadian oscillatory mechanism. Genes Dev 2001; 15(8):995-1006.

56. Miyazaki K, Kawamoto T, Tanimoto K, et al. Identification of functional hypoxia response elements in the promoter region of the DEC1 and DEC2 genes. J Biol Chem 2002;277(49):47014-21.

57. Noshiro M, Kawamoto T, Furukawa M, et al. Rhythmic expression of DEC1 and DEC2 in peripheral tissues: DEC2 is a potent suppressor for hepatic cytochrome P450s opposing DBP. Genes Cells 2004;9:317-29.

58. Bookout $A L$, Jeong $Y$, Downes $M$, et al. Anatomical profiling of nuclear receptor expression reveals a hierarchical transcriptional network. Cell 2006; 126:789-99.

59. Yang X, Downes M, Yu RT, et al. Nuclear receptor expression links the circadian clock to metabolism. Cell 2006;126:801-10.

60. Yang X. A wheel of time: the circadian clock, nuclear receptors, and physiology. Genes Dev 2010;24(8):741-7.

61. Galman C, Angelin B, Rudling M. Bile acid synthesis in humans has a rapid diurnal variation that is asynchronous with cholesterol synthesis. Gastroenterology 2005;129:1445-53.

62. Moore JT, Goodwin B, Willson TM, et al. Nuclear receptor regulation of genes involved in bile acid metabolism. Crit Rev Eukaryot Gene Expr 2002; 12:119-35.

63. Mazzoccoli G, Pazienza V, Vinciguerra M. Clock genes and clock controlled genes in the regulation of metabolic rhythms. Chronobiol Int 2012;29(3): 227-51.

64. Lavery DJ, Schibler U. Circadian transcription of the cholesterol 7alpha hydroxylase gene may involve the liver-enriched bZIP protein DBP. Genes Dev 1993;7:1871-84.

65. Wang $Y$, Kumar N, Solt LA, et al. Modulation of ROR \{alpha\} and ROR\{gamma\} activity by 7-oxygenated sterol ligands. J Biol Chem 2009;285:5013-25. 
66. Raspé E, Duez H, Gervois P, et al. Transcriptional regulation of apolipoprotein C-III gene expression by the orphan nuclear receptor RORalpha. J Biol Chem 2001;276(4):2865-71.

67. Raspé E, Duez H, Mansen A, et al. Identification of Rev-erbalpha as a physiological repressor of apoC-III gene transcription. J Lipid Res 2002;43: 2172-9.

68. Le Martelot G, Claudel T, Gatfield D, et al. REV-ERBalpha participates in circadian SREBP signaling and bile acid homeostasis. PLoS Biol 2009;7(9): e1000181.

69. Duez H, van der Veen JN, Duhem C, et al. Regulation of bile acid synthesis by the nuclear receptor Rev-erb $\alpha$. Gastroenterology 2008;135:689-98.

70. Gachon F, Nagoshi E, Brown SA, et al. The mammalian circadian timing system: from gene expression to physiology. Chromosoma 2004;113: 103-12.

71. Gatfield D, Schibler U. Circadian glucose homeostasis requires compensatory interference between brain and liver clocks. Proc Natl Acad Sci U S A 2008;105(39):14753-4.

72. Zhang EE, Liu Y, Dentin R, et al. Cryptochrome mediates circadian regulation of CAMP signaling and hepatic gluconeogenesis. Nat Med 2010;16(10): 1152-6.

73. Doi R, Oishi K, Ishida N. CLOCK regulates circadian rhythms of hepatic glycogen synthesis through transcriptional activation of Gys2. J Biol Chem 2010;285(29):22114-21.

74. Guillaumond F, Gréchez-Cassiau A, Subramaniam M, et al. Kruppel-like factor KLF10 is a link between the circadian clock and metabolism in liver. Mol Cell Biol 2010;30(12):3059-70.

75. Yamamoto T, Nakahata Y, Tanaka M, et al. Acute physical stress elevates mouse period1 mRNA expression in mouse peripheral tissues via a glucocorticoid- responsive element. J Biol Chem 2005; 280:42036-43.

76. So AY, Bernal TU, Pillsbury ML, et al. Glucocorticoid regulation of the circadian clock modulates glucose homeostasis. Proc Natl Acad Sci U S A 2009; 106(41):17582-7.

77. Yin L, Wu N, Curtin JC, et al. Rev-erbalpha, a heme sensor that coordinates metabolic and circadian pathways. Science 2007;318:1786-9.

78. Chauvet C, Vanhoutteghem A, Duhem C, et al. Control of gene expression by the retinoic acidrelated orphan receptor alpha in HepG2 human hepatoma cells. PLoS One 2011;6(7):e22545.

79. Tao W, Chen S, Shi G, et al. SWltch/sucrose nonfermentable (SWI/SNF) complex subunit BAF60a integrates hepatic circadian clock and energy metabolism. Hepatology 2011;54(4):1410-20.

80. Li S, Chen XW, Yu L, et al. Circadian metabolic regulation through crosstalk between casein kinase
$1 \delta$ and transcriptional coactivator PGC-1 $1 \alpha$. Mol Endocrinol 2011;25(12):2084-93.

81. Mazzoccoli G, Vinciguerra M, Oben J, et al. Nonalcoholic fatty liver disease: the role of nuclear receptors and circadian rhythmicity. Liver Int 2014; 34(8):1133-52.

82. Ando H, Takamura T, Matsuzawa-Nagata N, et al. Clock gene expression in peripheral leucocytes of patients with type 2 diabetes. Diabetologia 2009;52(2):329-35.

83. Dupuis J, Langenberg C, Prokopenko I, et al. New genetic loci implicated in fasting glucose homeostasis and their impact on type 2 diabetes risk. Nat Genet 2010;42(2):105-16.

84. Liu C, Li H, Qi L, et al. Variants in GLIS3 and CRY2 are associated with type 2 diabetes and impaired fasting glucose in Chinese Hans. PLoS One 2011; 6(6):e21464.

85. Rudic R, McNamara P, Curtis AM, et al. BMAL1 and CLOCK, two essential components of the circadian clock, are involved in glucose homeostasis. PLoS Biol 2004;2:e377.

86. Marcheva B, Ramsey KM, Buhr ED, et al. Disruption of the clock components CLOCK and BMAL1 leads to hypoinsulinaemia and diabetes. Nature 2010;466(7306):627-31.

87. Sadacca LA, Lamia KA, deLemos AS, et al. An intrinsic circadian clock of the pancreas is required for normal insulin release and glucose homeostasis in mice. Diabetologia 2011;54(1):120-4.

88. Pulimeno P, Mannic T, Sage D, et al. Autonomous and self-sustained circadian oscillators displayed in human islet cells. Diabetologia 2013;56(3):497-507.

89. Saini C, Petrenko V, Pulimeno P, et al. A functional circadian clock is required for proper insulin secretion by human pancreatic islet cells. Diabetes Obes Metab 2016;18(4):355-65.

90. Lamia KA, Storch KF, Weitz CJ. Physiological significance of a peripheral tissue circadian clock. Proc Natl Acad Sci U S A 2008;105(39):15172-7.

91. Targher G, Day CP, Bonora E. Risk of cardiovascular disease in patients with nonalcoholic fatty liver disease. N Engl J Med 2010;363:1341-50.

92. Williams CD, Stengel J, Asike MI, et al. Prevalence of nonalcoholic fatty liver disease and nonalcoholic steatohepatitis among a largely middle-aged population utilizing ultrasound and liver biopsy: a prospective study. Gastroenterology 2011;140(1): 124-31.

93. Bugianesi E, Leone N, Vanni E, et al. Expanding the natural history of nonalcoholic steatohepatitis: from cryptogenic cirrhosis to hepatocellular carcinoma. Gastroenterology 2002;123(1):134-40.

94. Podrini C, Borghesan M, Greco A, et al. Redox homeostasis and epigenetics in non-alcoholic fatty liver disease (NALFD). Curr Pharm Des 2013; 19(15):2737-46. 
95. Mazzoccoli G, Longhitano C, Vinciguerra M. Cardio-hepatic metabolic derangements and valproic acid. Curr Clin Pharmacol 2014;9(2):165-70.

96. Rotman Y, Sanyal AJ. Current and upcoming pharmacotherapy for non-alcoholic fatty liver disease. Gut 2017;66(1):180-90.

97. Sayin SI, Wahlström A, Felin J, et al. Gut microbiota regulates bile acid metabolism by reducing the levels of tauro-beta-muricholic acid, a naturally occurring FXR antagonist. Cell Metab 2013;17(2): 225-35.

98. Tremaroli V, Backhed F. Functional interactions between the gut microbiota and host metabolism. Nature 2012;489:242-9.

99. Cermakian N, Lange T, Golombek D, et al. Crosstalk between the circadian clock circuitry and the immune system. Chronobiol Int 2013;30(7):870-88.

100. Vinciguerra M, Borghesan M, Pazienza V, et al. The transcriptional regulators, the circadian clock and the immune system. J Biol Regul Homeost Agents 2013;27(1):9-22.

101. Mazzoccoli G, Sothern RB, Greco G, et al. Timerelated dynamics of variation in core clock gene expression levels in tissues relevant to the immune system. Int J Immunopathol Pharmacol 2011;24(4): 869-79.

102. Ma D, Panda S, Lin JD. Temporal orchestration of circadian autophagy rhythm by C/EBP $\beta$. EMBO J 2011;30(22):4642-51.

103. Muegge BD, Kuczynski J, Knights D, et al. Diet drives convergence in gut microbiome functions across mammalian phylogeny and within humans. Science 2011;332:970-4.

104. Chu H, Khosravi A, Kusumawardhani IP, et al. Gene-microbiota interactions contribute to the pathogenesis of inflammatory bowel disease. Science 2016;352(6289):1116-20.

105. Kim KH, Lee MS. Autophagy-a key player in cellular and body metabolism. Nat Rev Endocrinol 2014; 10(6):322-37.

106. Lee JM, Wagner M, Xiao R, et al. Nutrient-sensing nuclear receptors coordinate autophagy. Nature 2014;516(7529):112-5.

107. Seok S, Fu T, Choi SE, et al. Transcriptional regulation of autophagy by an FXR-CREB axis. Nature 2014;516(7529):108-11.

108. Madrigal-Matute J, Cuervo AM. Regulation of liver metabolism by autophagy. Gastroenterology 2016;150(2):328-39.

109. Zhang X, Han J, Man K, et al. CXC chemokine receptor 3 promotes steatohepatitis in mice through mediating inflammatory cytokines, macrophages and autophagy. J Hepatol 2016;64(1):160-70.

110. Bieghs V, Trautwein C. Innate immune signaling and gut-liver interactions in non-alcoholic fatty liver disease. Hepatobiliary Surg Nutr 2014;3(6): 377-85.
111. Kim S, Park S, Kim B, et al. Toll-like receptor 7 affects the pathogenesis of non-alcoholic fatty liver disease. Sci Rep 2016;6:27849.

112. Lin CW, Zhang H, Li M, et al. Pharmacological promotion of autophagy alleviates steatosis and injury in alcoholic and non-alcoholic fatty liver conditions in mice. J Hepatol 2013;58(5):993-9.

113. Mukherji A, Kobiita A, Ye T, et al. Homeostasis in intestinal epithelium is orchestrated by the circadian clock and microbiota cues transduced by TLRs. Cell 2013;153(4):812-27.

114. Clemente JC, Ursell LK, Parfrey LW, et al. The impact of the gut microbiota on human health: an integrative view. Cell 2012;148(6):1258-70.

115. Li F, Jiang C, Krausz KW, et al. Microbiome remodelling leads to inhibition of intestinal farnesoid $\mathrm{X}$ receptor signalling and decreased obesity. Nat Commun 2013;4:2384.

116. Tevy MF, Giebultowicz J, Pincus Z, et al. Aging signaling pathways and circadian clockdependent metabolic derangements. Trends Endocrinol Metab 2013;24(5):229-37.

117. Vinciguerra M, Tevy MF, Mazzoccoli G. A ticking clock links metabolic pathways and organ systems function in health and disease. Clin Exp Med 2014; 14(2): 133-40.

118. Jones ML, Tomaro-Duchesneau C, Prakash S. The gut microbiome, probiotics, bile acids axis, and human health. Trends Microbiol 2014;22(6):306-8.

119. Joyce SA, MacSharry J, Casey PG, et al. Regulation of host weight gain and lipid metabolism by bacterial bile acid modification in the gut. Proc Natl Acad Sci U S A 2014;111(20):7421-6.

120. Ridlon JM, Kang DJ, Hylemon PB, et al. Bile acids and the gut microbiome. Curr Opin Gastroenterol 2014;30(3):332-8.

121. Fiorucci S, Distrutti E. Bile acid-activated receptors, intestinal microbiota, and the treatment of metabolic disorders. Trends Mol Med 2015;21(11):702-14.

122. Flynn CR, Albaugh VL, Cai S, etal. Bile diversion to the distal small intestine has comparable metabolic benefits to bariatric surgery. Nat Commun 2015;6:7715.

123. Jiang C, Xie C, Li F, et al. Intestinal farnesoid X receptor signaling promotes nonalcoholic fatty liver disease. J Clin Invest 2015;125(1):386-402.

124. Lu P, Yan J, Liu K, et al. Activation of aryl hydrocarbon receptor dissociates fatty liver from insulin resistance by inducing fibroblast growth factor 21. Hepatology 2015;61:1908-19.

125. Vinciguerra M, Mazzoccoli G. Aryl hydrocarbon receptor-fibroblast growth factor 21 dissociation of fatty liver from insulin resistance: a timely matter? Hepatology 2016;63(4):1396-7.

126. Bookout AL, de Groot MH, Owen BM, et al. FGF21 regulates metabolism and circadian behavior by acting on the nervous system. Nat Med 2013; 19(9):1147-52. 